\title{
The Antigenic Specificity of the Humoral Immune Response to Primary and Repeated Ocular Infections of the Guinea Pig with the GPIC Agent (Chlamydia psittaci)
}

\author{
JOHN D. TREHARNE and ASAAD SHALLAL \\ London
}

\begin{abstract}
Summary
The antigenic specificity of the humoral immune response in guinea pigs to primary and repeated ocular infections with the guinea pig inclusion conjunctivitis (GPIC) chlamydial agent was analysed using microbiological, serological and Western blotting techniques. The results indicate that although there was a response to many minor polypeptide antigens, there was a marked lack of reactivity to the major outer membrane protein (MOMP), particularly following reinfection of guinea pigs. It is suggested that, lack of a good antibody response to the MOMP, may be one of the reasons why guinea pigs are susceptible to repeated ocular infections with this chlamydial agent.
\end{abstract}

It is generally acknowledged that trachoma is the most widespread preventable blinding eye disease in developing countries. ${ }^{1}$ Infection is caused by the obligate intracellular microorganism, Chlamydia trachomatis, serovars A, B, Ba and C. An interesting feature of trachoma is that re-infections or recurrences of infection occur and that there appears to be a short-lived or limited immunity to re-infection. ${ }^{2,3}$ Immunoprophylaxis has been shown to be ineffective, since the response to vaccine trials in humans using live chlamydial elementary bodies as the immunogen was inconsistent. Although a short-lived protective immunity was experienced in some cases, ${ }^{4,5}$ in the majority of cases there was a deleterious hypersensitivity response to vaccination. Recurrent genital tract infections caused by C. trachomatis serovars D-K have recently been reported and this may indicate that such recurrences are a characteristic common to all chlamydial infections. ${ }^{6,7}$

An essential prerequisite for the development of a successful vaccine strategy should be based on a thorough understanding of the cell-mediated and humoral immune response to chlamydial infection. The crucial role that chlamydial antigens play in eliciting the mechanisms of protective immunity also requires elucidation. Non-human primate and guinea pig animal models have been most widely used in advancing our knowledge in this area. In this study, guinea pigs were given primary and repeated ocular infections with the guinea pig inclusion conjunctivitis agent (GPIC) - a member of the Chlamydia psittaci species. The humoral immune response to infection was analysed, using serological and Western

From the Section of Virology, Department of Pathology, Institute of Ophthalmology, Judd Street, London WC1, UK.

Correspondence to: Dr J. D. Treharne, Section of Virology, Department of Pathology, Institute of Ophthalmology, Judd Street, London WC1H 9QS. 
blotting techniques, whilst the presence of the organism in the conjunctiva was detected using a fluorescein-labelled type-specific monoclonal antibody against GPIC agent.

\section{Materials and Methods}

The GPIC strain A-10 used for ocular inoculation was propagated in the yolk sac of 6-8 day-old embryonated hens' eggs, ${ }^{8}$ whilst mitomycin-C treated McCoy cell cultures were used to propagate the GPIC organisms used for sodium dodecyl sulphate- polyacrylamide gel (SDS-PAGE) separation. ${ }^{9}$ Purification of cell culture grown organisms was carried out using density-gradient centrifugation in 'Triosil' gradients. ${ }^{10}$

Dunkin-Hartley guinea pigs weighing 250300 grams were used in all experiments. Before infection, and at all subsequent examinations, conjunctival scrapings were collected from the eyes of every animal. ${ }^{8}$ Scrapings were stained using a monoclonal antibody directed against the $60 \mathrm{kDa}$ protein of the GPIC agent in an indirect fluorescent antibody test, to check for the presence of chlamydial inclusions or free elementary bodies (EBs) of the organism. Before infection and at every subsequent examination, blood was withdrawn from each guinea pig by the intra-cardiac route. For the primary infection, five guinea pigs were inoculated by instilling approximately $1000 \mathrm{ELD}_{50}$ of yolk sac grown GPIC agent into the right conjunctival sac. Collection of conjunctival scrapings and blood was carried out on days $0,3,5,7$, $10,14,21,28,35$ and 115 post-infection. These guinea pigs were reinfected with GPIC agent 115 days after the primary infection and collections were made from them on days 0 , $10,17,25,33$ and 415 after the first re-infection. A second reinfection of the guinea pigs was carried out 530 days after the primary infection and 415 days after the first reinfection, specimen collections being made on days $0,4,8,17$ and 240 . Whilst five guinea pigs were originally infected, unfortunately only three survived the rigors of the experimental procedures and were available for complete analysis at the end of the observation period.

Antibodies to the A-10 strain of GPIC agent were estimated using purified cell culture grown GPIC EBs in a modified microimmunofluorescence test (micro-IF). ${ }^{11}$
Two guinea pigs were hyperimmunised with Triosil $^{\mathrm{TM}}$ purified elementary bodies of the GPIC agent. This antigen was mixed with Freund's incomplete adjuvant and administered to the guinea pigs by multiple intracutaneous inoculations on days $0,21,42$ and 56 . On each occasion approximately $100 \mathrm{ug}$ of GPIC antigen (protein as measured by the Lowry assay), ${ }^{12}$ were injected into each guinea pig, both animals being exsanguinated on day 66.

Sodium dodecyl sulphate-polyacrylamide gel electrophoresis was carried out according to the procedure described by Laemmli (1970). ${ }^{13}$ After electrophoresis, the SDStreated GPIC antigens were electrophoretically blotted onto nitrocellulose sheets (NC) using the phosphate buffer of Bittner et al. ${ }^{14}$ Antigen-antibody reaction was performed essentially as previously described. ${ }^{15}$ Briefly, the blotted NC sheet was incubated for one hour at room temperature with BSA-GS-TN (bovine serum albumin 3\%[w/v], 5\% [v/v] normal goat serum and $0.1 \%$ sodium azide in $20 \mathrm{mM}$ Tris- $\mathrm{HCl} \mathrm{pH} \mathrm{7.4,} \mathrm{in} 150 \mathrm{mM} \mathrm{NaCl}$ ) to block the non-specific sites. The blocked NC sheet was then allowed to react first with guinea pig sera (diluted 1/35 in BSA-GS-TN) for four hours at room temperature and washed thoroughly with Tris-HCl-saline pH 7.4. Subsequently the NC sheet was incubated with peroxidase-conjugated anti-guinea pig whole immunoglobulins (Sigma Ltd) diluted with BSA-GS-TN for one hour at room temperature, and then washed again at least five times with Tris-HCl-saline. Antibody-antigen reaction was visualised by the addition of the substrate 4-chloro-1-naphthol (Sigma Ltd), as previously described. ${ }^{15}$

\section{Results}

Elementary bodies of Chlamydia psittaci strain GPIC were used to produce both primary and repeated ocular infections in guinea pigs clinically resembling the human disease trachoma which is caused by $C$. trachomatis $^{16}$ (Fig. 1). Chlamydial inclusions were detected using a fluorescein-conjugated monoclonal antibody produced against the $60 \mathrm{kDa}$ protein of the outer membrane of the GPIC chlamydial agent. The percentage of epithelial cells containing chlamydial 
inclusions was described as,,++++++ 'inclusion scores' which represent: less than $10 \%$; between $11-30 \%$ and more than $30 \%$, of the conjunctival epithelial cells having chlamydial inclusions. Figure 2 shows that more than $30 \%$ of the epithelial cells were infected by days five and seven of the primary infection. The percentage of infected epithelial cells was lower following the second and third ocular infections. It was also noted that the period during which inclusions could be detected in the conjunctivae was shorter in the second and third infections (17 and 10 days after each infection respectively), whereas inclusions could be detected up to 28 days following the primary infection.

The titre of the humoral immune response as judged by the micro-immunofluorescence secrology test is also shown in Figure 2. Following infection, the presence of serum antibodies to the GPIC agent in these guinea pigs was prolonged and persisted for many months. However, the identity and specificity of the reacting chlamydial antigens could only be identified by Western blotting (Fig. 3). Figure $3 \mathrm{~b}$ shows that blotted GPIC antigens could not be detected until the 21st day after the first (primary) infection when they were reacted with a pool of antisera collected from three infected guinea pigs. This correlates well with the high antibody titre seen in these sera at this time by the micro-IF test (Fig. 2). On day 21 , five prominent reactive antigens were detected by immunoblotting, representing proteins of apparent molecular weights of $93 \mathrm{kDa}, 60-62 \mathrm{kDa}, 39 \mathrm{kDa}$ [major outer membrane protein (MOMP)], $33 \mathrm{kDa}$ and the lipopolysaccharide (LPS) antigens. A similar reaction could still be seen on day 28 . With respect to these reactive antigens the response to the $60-62 \mathrm{kDa}$ protein was even stronger on day 115 after the primary infection. However, the only other antigens to which the immune response remained persistent on day 115 was the $93 \mathrm{kDa}$ antigen and to lesser extent, the LPS.

Following the second ocular infection, strong reaction to these five antigens was seen on day 17 (Fig. 3c). This is slightly earlier than in the first infection (21 days). The MOMP produced a strong reaction; there was also an antibody response to one further reactive antigen-18 kDa. Antibody response to some of these antigens persisted for 415 days following the second infection. LPS and the $33 \mathrm{kDa}$ protein (in animals 1 and 2) exhibited the most marked reactivity. Other rather fainter bands were also seen (Figure 3c).

The third ocular infection was carried out 530 days and 415 days after the first and second infections respectively (Fig. 3d). Antibodies to the $60-62 \mathrm{kDa}$ polypeptides were seen in all three animals with a fainter reaction to the MOMP after eight days. These reactions were stronger on day 15 , although the sera from only two animals showed intense bands representing the MOMP. Strong immuno-reactivity to the LPS and $33 \mathrm{kDa}$ species persisted before and after the third infection. Other minor reactive bands were also seen. On the other hand, pooled sera collected from two guinea pigs which had been parenterally hyperimmunised with GPIC agent over a period of two months, showed a strong immunoblotting reaction with seven antigens; LPS, $18 \mathrm{kDa}, 33 \mathrm{kDa}$, MOMP, 60-62 kDa, $84 \mathrm{kDa}$ and $93 \mathrm{kDa}$ (Fig. 3a [Lane $\mathrm{HI}]$ ).

\section{Discussion}

Epidemiological studies of trachoma in $\operatorname{man}^{2}$ and in the eyes of experimental monkeys, ${ }^{17,18}$ indicate that the severe progressive immunopathological disease involving pannus and scar formation occurs only after re-infection.

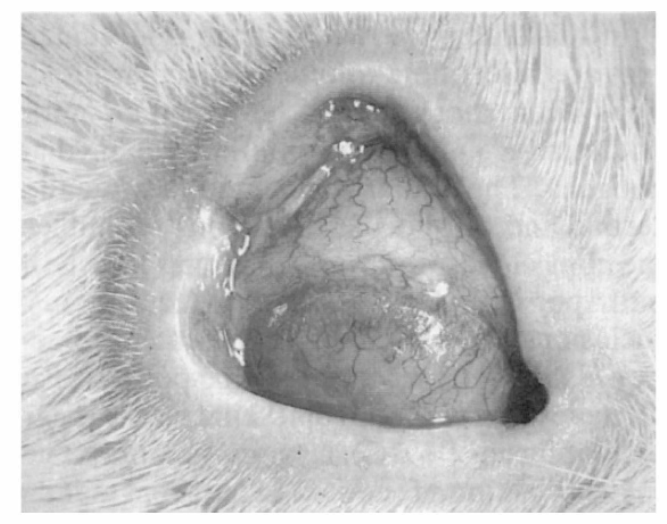

Fig. 1 Severe inflammatory response of guinea pig eye to re-infection with GPIC agent on Day 132 (i.e. Day 17 of 1 st reinfection). Note follicles and oedema of upper lid along with neovascularisation and infiltration of the cornea. 


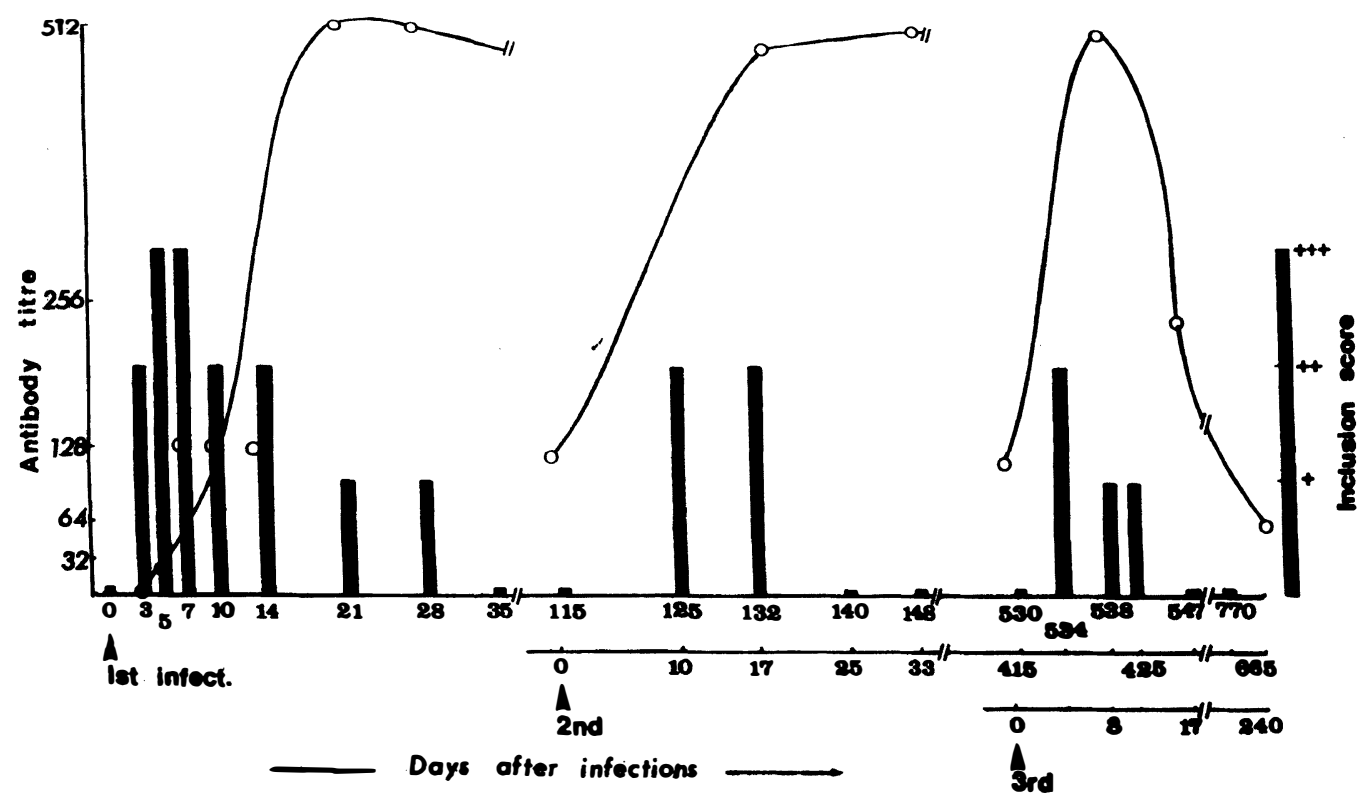

Fig. 2 Mean Inclusion scores and serological responses of guinea pigs in primary and re-infection experiments.

Susceptibility to re-infection was observed not only in ocular infection with Chlamydia trachomatis serovars $\mathrm{A}-\mathrm{C},{ }^{11}$ but also in urogenital infections caused by serovars $\mathrm{D}-\mathrm{K}^{19}$ and in experimental genital infection of guinea pigs with the GPIC agent of Chlamydia psittaci. $^{20}$ However, limited immunological protection was observed in guinea pigs that were injected with pooled immunoglobulins derived from other animals hyperimmunised with GPIC. ${ }^{21}$

In this study, we demonstrate that guinea pigs are susceptible to repeated ocular infections even though their sera contain antibodies to a number of different chlamydial antigens. The ocular inflammation after the second and third ocular infections resembles the clinical features seen in the first infection but persists for a shorter duration. ${ }^{16}$ Inclusions were present in repeated infections, although for a shorter time period; also a smaller percentage of epithelial cells appear to be infected (Fig. 2).

Examination of the sera taken from guinea pigs after the first ocular infection revealed the presence of persistent reactive antibodies to the $60-62 \mathrm{kDa}$ and, to lesser extent, a $93 \mathrm{kDa}$ protein. However, following the second ocular infection, antibodies to the LPS and the $33 \mathrm{kDa}$ antigens were more prominent up to day 415 , whereas there was considerably less response to the $60-62 \mathrm{kDa}$ and MOMP proteins. Antibodies to the $33 \mathrm{KD}$ and LPS antigens persist in sera for up to 300 days following the third infection (results not shown).

The most interesting finding in this study is the lack of a significant response to the MOMP protein after both the primary (acute) and repeated infections. This is similar to an earlier immunoblotting study of sera from patients with trachoma. ${ }^{10}$ The weak response is disproportionate to the amount of the MOMP, which represents about $60 \%$ of the total protein in the outer membrane of chlamydial EBs. It is also in contrast to the results obtained in experimental monkeys ${ }^{3}$ and in serum analysis of humans exposed to lymphogranuloma venereum. ${ }^{10}$ However, antisera obtained from sheep immunised with whole purified EBs of the sheep abortion agent of Chlamydia psittaci reacted strongly with the MOMP using the Western blotting technique, whereas only a weak reaction was seen in sera from sheep with the naturally occurring enzootic abortion infection. ${ }^{22}$ It is important to emphasise that in our experiments, the lack of strong cross-reactivity between the guinea 


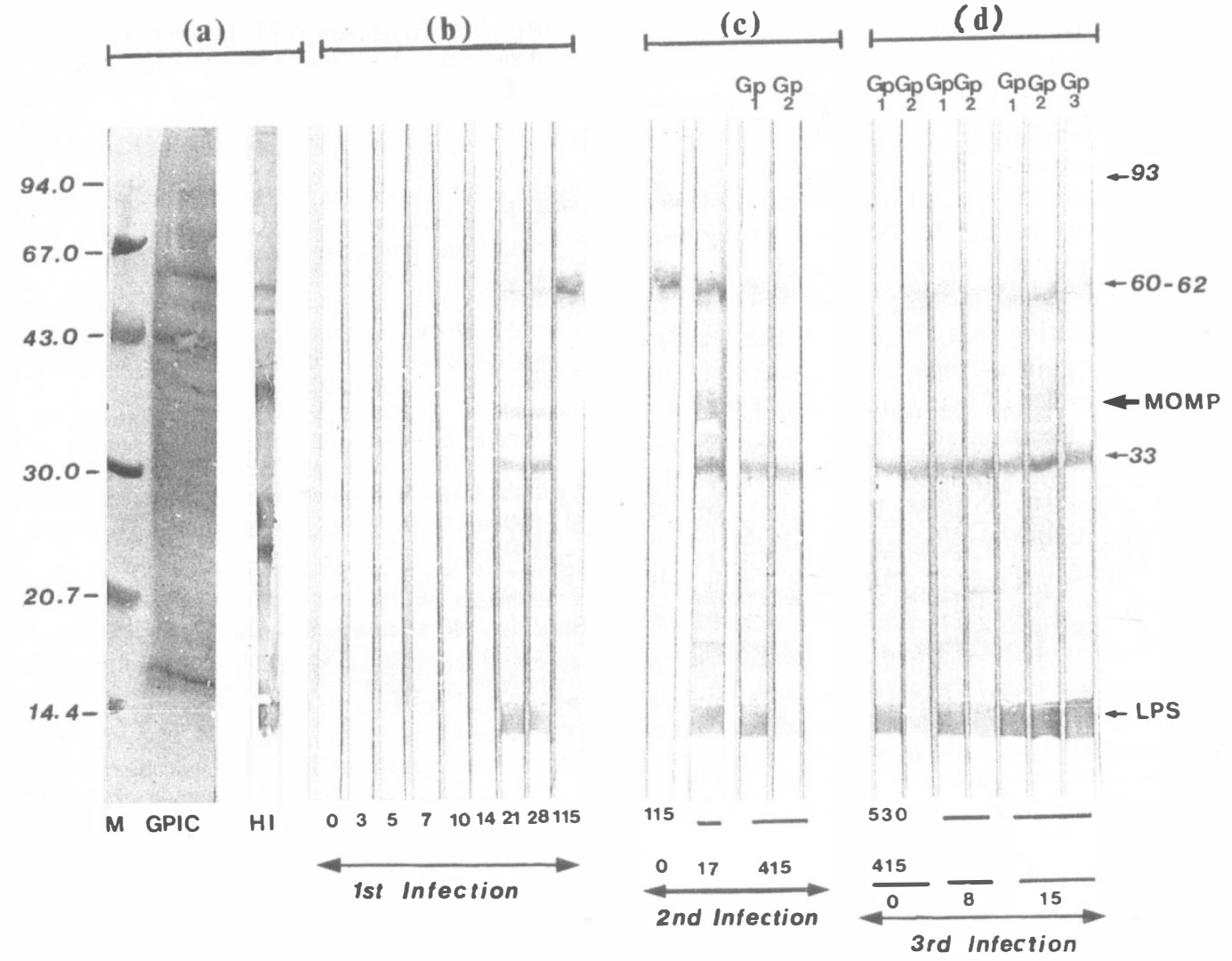

Fig. 3a-d. Immunoblotting (Western blotting) analysis following SDS-PAGE separation of guinea pig sera collected after primary ( $3 b)$ and repeated infections ( $3 c$ and $3 d)$ with GPIC agent.

Fig. 3a. M: $\quad$ represents protein molecular weight markers electro-blotted onto NC and stained with amido-black.

GPIC: $\quad$ is the protein profile of GPIC agent EBs following SDS-PAGE and electro-blotting, stained with amido-black.

HI: $\quad$ profile as above but proteins have been probed with hyperimmune anti-GPIC sera and visualised using the peroxidase technique as outline in the methods.

Fig. 3b. Immunoblots of pooled guinea pig sera collected during the primary infection.

Fig. 3c. Immunoblots of pooled sera collected on days 0 and 7 following the 2 nd infection. Immunoblots of sera of guinea pigs 1 and 2 are shown separately on day 415 .

Fig. 3d. Immunoblots of individual guinea pig sera collected during the third infection.

pig sera and the MOMP was not due to an inefficient transfer of the protein onto the nitrocellulose sheet; hyperimmune sera from other guinea pigs, which had been immunised intracutaneously with the GPIC agent, reacted strongly with the MOMP protein, as shown in Fig. 3a (Lane HI). This observation may reflect a hindrance of the MOMP's antigenic determinants of the GPIC agent due to the phenomenon of MOMP-LPS cross-linking, ${ }^{23}$ or it may be due to some other structural organisation in the outer membrane of chla- mydial EBs which enable the organism to evade the host's immune response. Alternatively, the immunodeterminant of the GPIC MOMP, recognised by guinea pig sera is conformation-dependent and that this determinant may have been muted or destroyed by SDS-PAGE separation and electroblotting. The fact that sera from hyperimmunised guinea pigs reacted strongly with MOMP may well reflect a difference in the immune response due to a different route of administration. Others have recently 
observed that subcutaneous immunisation of guinea pigs with purified GPIC SDS-MOMP complex does not induce any protective immune response, ${ }^{24}$ possibly indicating that SDS denatures protective immunogenic determinents on the MOMP. Since however, it is known that the MOMP is a strong neutralizing protein in vitro, ${ }^{25}$ lack of good immune response to this protein, as shown by immunoblotting, may be one of the reasons why the guinea pigs are susceptible to repeated cycles of ocular infection. Our findings may substantiate earlier studies ${ }^{10}$ on human sera collected from trachoma patients, using Western blotting techniques, which indicated that the chlamydial MOMP may have implications as an important candidate antigen for the future development of trachoma vaccines.

This work was generously supported by a research grant from the Trustees of Moorfield's Eye Hospital, London and has been reported as an extended abstract in 'Chlamydial Infections', eds. Bowie WR, Caldwell HD et al. Cambridge University Press, Cambridge, 1990, pp 261-264.

\section{References}

${ }^{1}$ Darougar S and Jones BR: Trachoma. Br Med Bull 1983;39:117-22.

${ }^{2}$ Grayston JT, Wang S-P, Yeh L-J, Kuo C-C: Importance of reinfection in the pathogenesis of trachoma. Rev Infect Dis 1985;7:717-25.

${ }^{3}$ Zang Y-X, Stewart S, Joseph T, Taylor HR,Caldwell HD: Protective monoclonal antibodies recognise epitopes located on the major outer membrane protein of $C$. trachomatis. J Immunol 1987;138:575-81.

${ }^{4}$ Grayston JT, Wooldridge RL, Wang S-P. Trachoma vaccine studies on Taiwan. Ann NY Acad Sci 1962;98:352-66.

${ }^{5}$ Jones BR: The prevention of blindness from trachoma. Trans Ophthalmol Soc UK 1975;95:16-33.

${ }^{6}$ Katz BP, Jones RB, Danos CS: Efficiency and costeffectiveness of field follow-up for patients with C. trachomatis infection in a STD clinic. In: Oriel D, Ridgway G, et al., eds. Chlamydial Infections. Cambridge: Cambridge University Press. 1986;495-8.

${ }^{7}$ Stamm WE, Roddy RE, Barnes RC: Repeated genital infections with $\mathrm{C}$. trachomatis: prevalence and risk factors. In: Oriel D, Ridgway G, et al., eds. Chlamydial Infections. Cambridge: Cambridge University Press. 1986;499-502.

${ }^{8}$ Treharne JD: A comparison of microbiological techniques for the detection of Gpic agent in the conjunctiva of infected guinea pigs. In: Nichols
RL, ed. Trachoma and Related Disorders. Amsterdam: Excerpta Medica. 1971;435-44.

${ }^{9}$ Woodland RM, Kirton RP, Darougar S: Sensitivity of mitomycin-C treated McCoy cells for isolation of C. trachomatis from genital specimens. Eur $J$ Clin Microbiol 1987;6:653-6.

${ }^{10}$ Ward ME, Treharne JD, Murray A: Antigenic specificity of human antibody to Chlamydia in trachoma and lymphogranuloma venereum. J Gen Microbiol 1986;132:1599-610.

${ }^{11}$ Treharne JD, Darougar S, Jones BR: Modification of the microimmunofluorescence test to provide a routine serodiagnostic test for chlamydial infection. J Clin Pathol 1977;30:510-7.

${ }^{12}$ Lowry OH, Rosebrough NJ, Farr AL, Randall RJ: Protein measurement with the folin phenol reagent. J Biol Chem 1951;193:265-75.

${ }^{13}$ Laemmli UK: Cleavage of structural proteins during assembly of the head of bacteriophage T4. Nature 1970;227:680-5.

${ }^{14}$ Bittner M, Kupferer P, Morris CF: Anal Biochem 1980;102:459-71.

${ }^{15}$ Shallal A, Al-Mahdawi S, Kolodzeig M, Converse CA: A specific ELISA using purified opsin for studying autoimmunity in retinal diseases. $J$ Clin Lab Immunol 1988;27:45-50.

${ }^{16}$ Monnickendam MA, Darougar S, Treharne JD, Tilbury AM: Development of chronic conjunctivitis with scarring and pannus resembling trachoma in guinea pigs. Br J Ophthalmol 1980;64: 284-90.

${ }^{17}$ Wang S-P and Grayston JT: Pannus with experimental trachoma and inclusion conjunctivitis agent infection of Taiwan monkeys. Am J Ophthalmol 1967;63:1133-45.

${ }^{18}$ Taylor HR, Johnson SL, Schachter J, Caldwell HD, Prendergast RA: Pathogenesis of trachoma: The stimulus for inflammation. J Immunol 1987;138:3023-7.

${ }^{19}$ Rank RG, Batteiger BE, Soderberg LSF: Susceptibility to reinfection after a primary chlamydial genital infection. Infect Immun 1988;56:2243-49.

${ }^{20}$ Batteiger BE, Rank RG: Analysis of the humoral immune response in chlamydial genital infection in guinea pigs. Infect Immun 1987;55:1767-73.

${ }^{21}$ Rank RG and Batteiger BE: Protective role of serum antibody in immunity to chlamydial genital infection. Infect Immunol 1989;57:299-301.

${ }^{22}$ Tan TW, Herring AJ, McClenaghan M, Huang H-S et al. Immunoblotting analysis of the humoral immune response in sheep infected with the ovine abortion strain of C. psittaci In: Proceedings of the European Society of Chlamydia Research. Bologna: Societa Editrice Esculapio. 1988;p. 136.

${ }^{23}$ Birkeland S, Lundemose AG, Christiansen G: Chemical cross-linking of Ctrachomatis. Infect Immun 1988;56:654-9.

${ }^{24}$ Batteiger BE, Rank RG, Soderberg LS: Immunization of guinea pigs with isolated chlamydial outer membrane proteins. In, Chlamydial Infections, Eds Bowie WR, Caldwell HD, et al. Cambridge University Press, Cambridge, 1990 pp 265-268.

${ }^{25}$ Caldwell HD, Perry L: Neutralization of C. trachomatis infectivity with antibodies to the major outer membrane protein. Infect Immun 1982;38:745-54. 